\title{
Risk Factors Associated with Arterial Stiffness in Diabetic Nephropathy in an Asian Population Cohort
}

\author{
Lim Su Chi², Phua E Joo², Sharon F Nne', Amizah B Asrap³, Wong DS Melvin ${ }^{3}$, Tan SH Clara ${ }^{3}$, \\ Liu J Jun³, Toy W Ching 3 , Ng X Wei3, Lau PX Dawn³, Pek LT Sharon³, Woon Kaing3, \\ Lin LF Bernice3, S Tavintharan², Sum C Fang2, \\ ${ }^{1}$ Diabetes Clinic, Khoo Teck Puat Hospital, Singapore \\ ${ }^{2}$ Department of Medicine, Khoo Teck Puat Hospital, Singapore \\ ${ }^{3}$ Clinical Research Unit, Khoo Teck Puat Hospital, Singapore
}

\begin{abstract}
Introduction. Individuals with diabetic nephropathy (DN) are at risk for cardiovascular disease. Arterial stiffness summarizes an individual's global cardiovascular risk burden. We investigated the relationship between major modifiable metabolic risk factors (excluding blood pressure) and arterial stiffness DN.

Methods. Cross sectional study of 353 Diabetic Asians with Modified Diet to retard Renal Disease (MDRD) formula estimated glomerular filtration rate (eGFR) $<90 \mathrm{mls} / \mathrm{min} / 1.73 \mathrm{~m}^{2}$. Central Aortic Systolic Pressure (CASP), a surrogate measurement of arterial stiffness, was estimated using validated BPro A-Pulse ${ }^{\text {TM }}$ tonometry. Visceral fat area (VFA) was estimated by tetrapolar multi-frequency bio-impedence.

Results. Study population: $61 \%$ male, $9 \%$ current smoker, $84 \%$ receiving lipid lowering therapy and $45 \%$ taking insulin. Mean age(SD) was 61(9) year, CASP 130.0(21.4) mmHg, HBA1c 8.1(1.7)\%, eGFR 50.1(26.8) mls/min/1.73m², urinary albumin-creatinine ratio (ACR) 662(1339) $\mathrm{mg} / \mathrm{g}$, LDL 2.67(0.92) mM, VFA $130.0(31.9) \mathrm{cm}^{2}$. After adjusting for age, gender, ethnicity and smoking status, eGFR ( $\beta$ coefficient=-0.13) and HBA1c (1.66) remained as significant independent predictors of CASP $(\mathrm{P}<0.05)$. However, all the tested modifiable risk factors collectively explained only $\sim 10 \%$ of variation in CASP.

Conclusion. GFR and HBA1C are modifiable predictive factors for arterial stiffness in DN. However, our results suggested the presence of undiscovered novel risk factors.
\end{abstract}

Keywords: Diabetic nephropathy, arterial stiffness, central aortic systolic pressure.

\section{Introduction}

Asian diabetic individuals are highly susceptible to nephropathy ${ }^{1}$. Diabetic nephropathy $(\mathrm{DN})$ is also associated with increased risk for cardiovascular diseases $(\mathrm{CVD})^{2}$. The mechanism for CVD is likely to be complex and many of the risk factors may be potentially modifiable. These include excessive adiposity (in particular, visceral adiposity), glycemic burden, hypertension, dyslipidemia and deranged bone mineral metabolism ${ }^{3}$. These hemodynamic and metabolic factors lead to impaired endothelial function and vascular injury such as arterial stiffness ${ }^{4}$.

Several methods have been described to estimate arterial stiffness with pulse wave velocity (PWV) being considered as "gold standard". ${ }^{5}$ Alternatively, central aortic systolic pressure (CASP) is also considered a good surrogate index of arterial stiffness ${ }^{6}$. Recent reports demonstrated that noninvasively measured radial artery pressure waveform

ISSN 0857-1074

Copyright $(2011$ by the JAFES

Received August 3, 2011. Accepted October 5, 2011. can accurately estimate CASP in human ${ }^{7}$. These advances provided safe and reliable method to investigate vascular biology in vivo.

Diabetes mellitus is associated with arterial stiffness ${ }^{8}$. However, to the best of our knowledge, little is known about the risk factors (especially those amenable to interventions) associated with arterial stiffness in the subpopulation of DN subjects who are at extremely high risk for $\mathrm{CVD}^{9}$. Therefore, we investigated the relationship between potentially modifiable metabolic risk factors and arterial stiffness in a large group of Asian diabetic individuals with a wide range of renal filtration function.

\section{Methods}

\section{Patient population}

Convenient sampling of 577 consecutive diabetic individuals attending a single hospital-based diabetes 
centre. Diabetes mellitus was diagnosed according to World Health Organization proposed criteria. Subjects with Modified Diet to retard Renal Disease (MDRD) formula estimated glomerular filtration rate (eGFR) $<90$ $\mathrm{mls} / \mathrm{min} / 1.73 \mathrm{~m}^{2}$ (corresponding to stage 2 or more severe) were considered as having chronic kidney disease (CKD) ( $\mathrm{N}=353,61 \%$ of the overall study population). We defined $\mathrm{CKD}$ in this manner for the following reasons. Firstly, MDRD formula performs well when eGFR is $<90$ $\mathrm{mls} / \mathrm{min} / 1.73 \mathrm{~m}^{2}$ among diabetic individuals ${ }^{10}$. We did not choose the recently developed CKD-EPI formula because investigators reported that it did not perform better than simpler MDRD formula in diabetic population ${ }^{11}$. Secondly, when eGFR is $<90 \mathrm{mls} / \mathrm{min} / 1.73 \mathrm{~m}^{2}$, prevalence of comorbidities associated with CKD (e.g. hypertension) began to increase markedly suggesting that GFR at this level (or below) is associated with meaningful derangement in renal physiology $y^{12}$. Current smoker referred to subjects who have been smoking cigarettes daily for 3 months or more just prior to enrolment into the study.

\section{Central Aortic Systolic Pressure (CASP)}

BPro A-Pulse ${ }^{\mathrm{TM}}$ (Healthstat, Singapore) noninvasive tonometric device was used to measure CASP based on radial arterial waveform analysis. In this method, the radial waveform is calibrated to brachial blood pressure, measured using standard sphygmomanometry, thereby generating a calibrated radial artery pressure waveform (RAPWF). Using an n-point moving average (NPMA), a mathematical modeling method that acts as a low pass filter to smooth signal data to better determine underlying trends, investigators recently reported that RAPWF analysis could accurately estimate CASP in human ${ }^{13}$.

\section{Visceral fat area (VFA)}

Inbody S20 (Derwent Healthcare, Newcastle upon Tyne, United Kingdom) tetrapolar multi-frequency bioimpedence method was used to estimate VFA. Validated against VFA derived from computed tomography (CT) at the umbilical level, this convenient method has been reported to accurately estimating $\mathrm{VFA}^{14}$. We decided to assess adiposity by measuring VFA in preference over body mass index (BMI) for the following reasons. Firstly, Asians are known to accumulate more body fat at any given BMI. Secondly, epidemiological data suggested visceral adiposity might confer greater burden of cardiovascular dysmetabolism than global obesity. Thirdly, patients with advance CKD may have protein malnutrition and hence sarcopenic. Therefore, their BMI may be low but paradoxically their CVD risk remains elevated. Hence, directly estimating adiposity using VFA may avoid this paradox when studying CKD patients.

\section{Clinical and biochemical parameters}

Anthropometric data were measured for all individuals. Two readings of blood pressure were taken from participants after five minutes resting using an automated blood pressure monitor (Dinamap Pro100V2; Criticon, Norderstedt, Germany) by trained observers. A third reading was performed if difference between two readings of systolic blood pressure (SBP) was $>10 \mathrm{mmHg}$ or diastolic blood pressure (DBP) was $>5 \mathrm{mmHg}$. The mean values of the closest two readings were calculated. Early morning spot urine sample was collected for albumin and creatinine measurement (albumin over creatinine ratio, ACR) using commercial assay (Immulite, DPC, Gwynedd, United Kingdom) with a lower detection limit of $6 \mathrm{mg} / \mathrm{L}$. Venous blood samples (taken after a 10-hour fast) with EDTA as anticoagulant were kept in icebox immediately after collection and the plasma was separated from erythrocytes by centrifuging at $1500 \mathrm{~g}$ for $10 \mathrm{~min}$ at $4^{\circ} \mathrm{C}$. Plasma creatinine levels were measured by means of Jaffé's kinetic method using a Roche Integra 800 analyzer. Blood lipids [Total cholesterol (TC), triglyceride (TG) and high density lipoprotein (HDL-C)] were measured by enzymatic methods using Kodak Ektachem chemistry slides, which were then read on a Vitros 700 Chemistry Analyser. HDL-C was measured after precipitation with dextran sulphate and magnesium chloride. LDL-C was calculated using Friedewald's formula.

\section{Statistical analysis}

Data are expressed as mean \pm SD. All statistical analysis was calculated using SPSS version 19.0. Chi-square analysis was used to test for difference in categorical variables. Student's $t$ test was employed to compare continuous variables between two groups. Analysis of variance (ANOVA) was used to compare continuous variables between three or more groups. To explore factors potentially predictive of variation in CASP, bi-variate correlational analysis was performed using Pearson Correlation (for normally distributed data) or Spearman's rho method (for non-normally distributed data). Factors significantly correlated with CASP $(\mathrm{P}<0.05)$ i.e. eGFR and urinary ACR were further tested in multi-variate analysis. General liner model (GLM) was used in multi-variate analysis. Though non-modifiable, the following factors known to influence CASP i.e. age, gender, ethnicity and smoking status were included in the model. Based on biological and clinical considerations, the following factors were also preferentially included i.e. HBA1c, LDLcholesterol, VFA. Blood pressure was excluded in the final model because BPro A-Pulse ${ }^{\mathrm{TM}}$ estimate CASP based on RAPWF was calibrated using brachial blood pressure. Therefore, CASP in our data expectedly showed unacceptably high co-linearity with systolic blood pressure $(\mathrm{SBP})(\mathrm{r}=0.87, \mathrm{P}<0.001)$ akin to circular reasoning. In fact, when SBP was included in the model, the overall correlation (R) was inflated to 0.92 suggesting over-fitting (detailed data not shown). Hence, the following modifiable and non-modifiable risk factors were included in our final multi-variate model: Gender, ethnicity, smoking status, age, eGFR, urinary ACR, HBA1c, LDL- 
cholesterol and VFA. A two-sided p-value was used and a p-value of $<0.05$ was considered statistically significant.

The study was approved by our center's Institution Review Board (IRB) and written informed consent was obtained from all participants.

\section{Results}

Among the 353 DN subjects, 94\% were type 2 diabetes, $60.6 \%$ were male; $62.6 \%$ Chinese, $27.8 \%$ Malay and $9.6 \%$ Indian; $9.4 \%$ were current smokers, $84 \%$ were receiving lipid lowering therapy, $45 \%$ were treated with insulin, $76 \%$ were treated with either angiotensin-convertingenzyme (ACE) inhibitor or angiotensin receptor blocker (ARB) or both. Clinical and laboratory data of the $353 \mathrm{DN}$ subjects are shown in table 1 . Most of the study subjects had moderately severe CKD with eGFR averaged at only $50.1(26.8) \quad \mathrm{mls} / \mathrm{min} / 1.73 \mathrm{~m}^{2} \quad$ (range: 2.8 to 89.9 ) corresponding to stage $3 \mathrm{CKD}$. There was no difference in CASP between gender, ethnic groups, current smoker vs. non-smoker. Therefore, CASP: male versus female [mean(SD] was 128.9(20.9) $\mathrm{mmHg}$ vs. 131.8(22.1) $(\mathrm{P}=0.22)$; Chinese versus Malay versus Indians was 128.8(20.6) $\mathrm{mmHg}$ vs. $134.6(23.8)$ vs. $124.5(16.8)$ ( $\mathrm{P}$ trend=0.12); Current smoker versus non-smoker was $131.0(21.5) \mathrm{mmHg}$ vs. 124.9(17.6) ( $P$ trend=0.11).

Table 1. Clinical and biochemical profile of subjects with diabetic nephropathy $(\mathrm{N}=353)$

\begin{tabular}{|c|c|}
\hline & Mean (SD) \\
\hline Age (years) & $61(10)$ \\
\hline Body mass index $\left(\mathrm{kg} / \mathrm{m}^{2}\right)$ & $27.7(8.9)$ \\
\hline Systolic blood pressure $(\mathrm{mmHg})$ & $141(20)$ \\
\hline Diastolic blood pressure $(\mathrm{mmHg})$ & $79(11)$ \\
\hline HBA1c (\%) & $8.1(1.7)$ \\
\hline Total cholesterol (mM) & $4.58(1.20)$ \\
\hline HDL-cholesterol (mM) & $1.19(0.38)$ \\
\hline LDL-cholesterol (mM) & $2.67(0.92)$ \\
\hline Triglyceride (mM) & $2.04(1.63)$ \\
\hline Glomerular filtration rate $\left(\mathrm{ml} / \mathrm{min} / 1.73 \mathrm{~m}^{2}\right)$ & $50.1(26.8)$ \\
\hline Urinary albumin/creatinine ratio $(\mathrm{mg} / \mathrm{g})$ & $662(1339)$ \\
\hline Visceral fat area $\left(\mathrm{cm}^{2}\right)$ & $130.0(31.9)$ \\
\hline Percentage body fat (\%) & $24.7(9.6)$ \\
\hline Central aortic systolic pressure $(\mathrm{mmHg})$ & $130.0(21.4)$ \\
\hline
\end{tabular}

When CASP is treated as a continuous variable, bi-variate correlational analysis is shown in table 2a. Only eGFR and urinary ACR were significantly correlated with CASP. Given that BPro A-Pulse ${ }^{\mathrm{TM}}$ estimated CASP was derived from RAPWF calibrated with brachial blood pressure, as expected, our data revealed extremely strong correlation between CASP and SBP. Therefore, as explained above, blood pressure was excluded from multi-variate analysis. Normative data of CASP is yet to be established. Therefore, when the normally-distributed CASP is treated as a categorical variable, dichotomized into two groups according to the mean value of $130 \mathrm{mmHg}$, comparison of risk factors between the two groups is as shown in table 2b. Subjects with high CASP were older $(\mathrm{P}=0.036)$, had high systemic blood pressure $(\mathrm{P}<0.001)$ and carried greater visceral adiposity $(\mathrm{P}=0.018)$.
Table 2a. Bi-variate correlation analysis between central aortic systolic pressure (CASP) and risk factors.

\begin{tabular}{lcc} 
& $\begin{array}{c}\text { Coefficient of } \\
\text { correlation }(\mathbf{r})\end{array}$ & $\begin{array}{c}\text { P value } \\
\text { (2-tailed) }\end{array}$ \\
\hline Age & 0.096 & 0.075 \\
Body mass index & -0.13 & 0.814 \\
Systolic blood pressure & 0.87 & $<0.001$ \\
Diastolic blood pressure & 0.43 & $<0 . .001$ \\
HBA1c & 0.05 & 0.39 \\
Total cholesterol & 0.07 & 0.29 \\
HDL-cholesterol & 0.06 & 0.25 \\
LDL-cholesterol & 0.06 & 0.28 \\
Triglyceride & 0.02 & 0.65 \\
Glomerular filtration rate & -0.15 & 0.005 \\
Urinary albumin/creatinine ratio & 0.13 & 0.023 \\
Visceral fat area & -0.008 & 0.88 \\
Percentage body fat & 0.024 & 0.66 \\
\hline
\end{tabular}

Table 2b. Clinical and biochemical profile of diabetic nephropathy subjects stratified by study-cohort mean central aortic systolic pressure (CASP) of $130 \mathrm{mmHg}$

\begin{tabular}{|c|c|c|c|}
\hline & $\begin{array}{c}\text { CASP }<130 \\
\mathrm{mmHg}\end{array}$ & $\begin{array}{c}\text { CASP } \geq 130 \\
\mathrm{mmHg}\end{array}$ & $P$ value \\
\hline Age (years) & $60(10)$ & $62(9)$ & 0.036 \\
\hline Body mass index $\left(\mathrm{kg} / \mathrm{m}^{2}\right)$ & $27.8(11.4)$ & $27.7(4.9)$ & 0.91 \\
\hline $\begin{array}{l}\text { Systolic blood pressure } \\
\text { (mmH) }\end{array}$ & $127(13)$ & 157(16) & $<0.001$ \\
\hline $\begin{array}{l}\text { Diastolic blood pressure } \\
\text { (mmHg) }\end{array}$ & $76(10)$ & $85(11)$ & $<0.001$ \\
\hline HBA1c (\%) & $8.3(1.8)$ & $8.3(1.7)$ & 0.21 \\
\hline Total cholesterol (mM) & $4.65(1.51)$ & $4.6(1.25)$ & 0.92 \\
\hline HDL-cholesterol (mM) & $1.23(0.40)$ & $1.21(0.34)$ & 0.54 \\
\hline LDL-cholesterol (mM) & $2.67(0.80)$ & $2.73(0.99)$ & 0.44 \\
\hline Triglyceride (mM) & $2.14(4.27)$ & $2.14(2.15)$ & 0.98 \\
\hline $\begin{array}{l}\text { Glomerular filtration rate } \\
\left(\mathrm{ml} / \mathrm{min} / 1.73 \mathrm{~m}^{2}\right)\end{array}$ & $89.6(128.9)$ & 83.3(134.8) & 0.56 \\
\hline $\begin{array}{l}\text { Urinary albumin/creatinine } \\
\text { ratio }(\mathrm{mg} / \mathrm{g})\end{array}$ & $388(1152)$ & $564(1150)$ & 0.09 \\
\hline Visceral fat area $\left(\mathrm{cm}^{2}\right)$ & $124.6(35.0)$ & $131.4(32.3)$ & 0.018 \\
\hline Percentage body fat (\%) & $24.4(10.1)$ & $25.7(9.5)$ & 0.11 \\
\hline
\end{tabular}

Table 3. Multi-variate analysis of predictors of central aortic systolic pressure.

\begin{tabular}{lcccc} 
& $\boldsymbol{\beta}$ & $\mathbf{P}$ value & $\boldsymbol{\beta}_{1}{ }^{*}$ & $\mathbf{P}$ value \\
\hline Constant & 138.5 & $<0.001$ & 128 & $<0.001$ \\
Age & 0.20 & 0.149 & 0.185 & 0.19 \\
Glomerular filtration rate & -0.145 & 0.021 & -0.127 & 0.045 \\
Albumin/creatinine ratio & 0.002 & 0.167 & 0.002 & 0.125 \\
HBA1c & 1.79 & 0.013 & 1.66 & 0.024 \\
LDL-cholesterol & 0.529 & 0.693 & 0.497 & 0.719 \\
Visceral fat area & 0.002 & 0.954 & 0.004 & 0.923 \\
\hline${ }^{*}$ Adjusted for gender, ethnicity and smoking status & &
\end{tabular}

Results of multi-variate analysis are shown in Table 3. Given that our intention was to focus on major modifiable risk factors for CASP and taking into consideration factors known to affect CASP (e.g. age and smoking status), the following were included in the final model: HBA1c, eGFR, ACR, LDL-cholesterol, VFA, age, gender, ethnicity and smoking status. Our results revealed that HBA1c and eGFR were significant independent predictors of CASP $(\mathrm{P}<0.05)$. This relationship was only modestly attenuated when additionally adjusted for gender, ethnicity and smoking status. However, when all the major modifiable risk factors (i.e. HBA1c, eGFR, ACR, LDL, VFA and smoking status) were considered together, they collectively only explained $\sim 10 \%$ of CASP variation in this group of DN subjects. 


\section{Discussion}

In a large, multi-ethnic Asian population with $\mathrm{DN}$, we demonstrated that renal glomerular filtration function and glycemic burden were important independent predictors of arterial stiffness (i.e. CASP). However, the major modifiable risk factors studied collectively accounted for only $\sim 10 \%$ of variation in CASP. This suggested the presence of undiscovered novel risk factors which may be amenable to interventions. Future study to uncover these risk factors may reveal novel therapeutic targets.

DN is associated with systemic vascular dysfunction manifesting as arterial stiffness which appears to progress in tandem with severity of $\mathrm{CKD}^{15}$. Therefore, it was perhaps not surprising that our data revealed CASP varied inversely with eGFR, i.e. increasing arterial stiffness with worsening renal filtration function. The mechanistic link between renal dysfunction and arterial stiffness is likely to be complex - there are direct and indirect processes. For instance, renal dysfunction is associated with chronic low grade inflammation ${ }^{16}$, activation of rennin-angiotensin aldosterone system ${ }^{17}$, increased oxidative stress, abnormal tubulo-glomerular feedback ${ }^{18}$ and deranged bone mineral metabolism ${ }^{19}$. These factors are known to be associated with vascular dysfunction ${ }^{20}$. Alternatively, arterial stiffness and renal dysfunction may share a "common soil" 21 . There is a growing body of evidence to suggest that components of cardiovascular dysmetabolism not only drive vascular injury but also predispose a person to $\mathrm{CKD}^{22}$. Corollary to this, interventions targeted at reducing cardiovascular burden are often reno-protective and vice versa ${ }^{23}$. Therefore, it appears reasonable to consider preservation of renal function as beneficial to prevent arterial stiffness.

Glycemic burden is another purported candidate for renovascular injury ${ }^{24}$. The mechanisms have been elegantly summarized by Brownlee et $\mathrm{al}^{25}$. Briefly, hyperglycemia is known to drive oxidative stress which leads to diversion of metabolic intermediary towards alternative "shunt pathways" such as polyol pathway, hexo-amine pathway, production of advance glycation end-product and activation of protein kinase $\mathrm{C}$. However, the efficacy of anti-hyperglycemic interventions in ameliorating CVD appeared somewhat difficult to prove in clinical trials ${ }^{26}$. Nevertheless, a few recent meta-analyses lend support to the cardiovascular benefits of reducing glycemic burden ${ }^{27}$. Therefore, optimization of glycemic control remains the cornerstone of diabetes management for the prevention of diabetic vascular complications.

In our cohort of DN patients, we did not observe any association between LDL-cholesterol and CASP. This is consistent with observations from clinical trials in which lipid lowering therapy among individuals on hemodialysis over a period of 3.8 years had no significant effect on fatal or non-fatal $\mathrm{CVD}^{28}$. Nevertheless, lipid lowering therapy continued to hold considerable promise as very recent clinical study reported significant reduction in cardiovascular endpoints among individuals with both diabetes and non-diabetes associated CKD ${ }^{29}$. Similarly, we did not observe any association between severity of albuminuria, visceral adiposity and CASP. Although albuminuria has been widely reported as a risk marker for CVD ${ }^{30}$, interventional studies successful in achieving significant anti-proteinuria could not uniformly demonstrate a reduction in cardiovascular events ${ }^{31}$. Having said so, in the PREVEND Intervention Trial (PREVEND IT), patients with albuminuria treated with fosinopril did report a trend toward a decrease in cardiovascular events ${ }^{32}$. Therefore, intervention targeted at albuminuria reduction remains a promising therapeutic strategy. As far as we know, there are no high quality clinical trials conducted to specifically test whether interventions targeted at reducing visceral adiposity could ameliorate vascular injury among DN subjects ${ }^{33}$. Taken together, our data supported the notion that conventional major cardiovascular risk factors (e.g. hyperlipidemia, albuminuria and visceral adiposity) might not fully explain the cardiovascular burden in diabetic individuals with $\mathrm{CKD}^{34}$. Much needs to be done to better understand the patho-biology behind CKD and CVD. It is thus important to search for novel cardiovascular risk factors (e.g. CKD-metabolic bone disease, adrenomedullin and adiponectin) in this high risk population.

The strength of our study included large, multi-ethnic, high risk (diabetic Asians) population which is largely under-represented in the literature. We used a validated method (BPro A-Pulse ${ }^{\mathrm{TM}}$ ) to measure CASP which is an accepted surrogate measurement of arterial stiffness. In addition, we measured visceral adiposity (i.e. VFA) rather than simpler but non-specific measurement of global obesity (i.e. BMI) in this group of CKD subjects at risk of protein malnutrition. There are however several limitations in our study. Firstly, this is a cross-section ecological study thereby precluding causal inference between risk factors studied and CASP. Therefore, our observations can only be considered as hypothesisgenerating. Secondly, we could not include SBP, a major risk factor for vascular injury, in our analysis given that CASP measurement was derived from SBP based on blood pressure calibrated RAPWF. This might have been possible if we had employed PWV ("gold standard") to estimate arterial stiffness. Thirdly, we did not systematically collect high quality information on concomitant medications i.e. type of drugs exposed, dosage and duration exposed etc. As a result, we were not able to account for this when performing statistical adjustment. Fourthly, our study did not include other promising risk markers for arterial stiffness in CKD population e.g. vitamin D. Therefore, our future plan is to study the relationship between traditional and novel risk factors and arterial stiffness (measured by PWV) in a large cohort of Asian diabetic individuals. 
In summary, eGFR and HBA1c are important modifiable risk factors for CASP in individuals with DN. However, the role of SBP on arterial stiffness in DN subjects deserves further study. Moreover, our results suggested possible presence of undiscovered novel risk factors which may be amenable to interventions.

\section{Acknowledgment}

Our most sincere thanks also go to the participants of this study for without their support this study would not have been possible.

\section{Source of funding}

This work was supported by grants from the Alexandra Health Small Innovative Grant II (SIGII/08005); National Medical Research Council (NMRC), Program Project Grant 2011-2012, Republic of Singapore and NMRC Enabling Grant to Alexandra Health 2008, 2009 and 2010.

The sponsor has no role in any of the following: design and conduct of the study; collection, management, analysis, and interpretation of the data; and preparation, review, or approval of the manuscript.

The corresponding author had full access to all of the data in the study and takes responsibility for the integrity of the data and the accuracy of the data analysis

\section{References}

1. Karter A, Ferrara A, et al. Ethnic disparities in diabetic complications in an insured population. JAMA. 2002; 287:2519-2527.

2. Sasso, FC. Cardiovascular risk factors and disease management in type 2 diabetic patients with diabetic nephropathy. Diabetes Care. 2006;29:498-503.

3. Cravedi P and Remuzzi G. Treating the kidney to cure the heart. Kidney International.2008;74(111):S2-S3.

4. Nakagawa T, Tanabe K, Croker BP, et al. Endothelial dysfunction as a potential contributor in diabetic nephropathy. Nat. Rev. Nephrol. 2011;7:36-44.

5. Laurent S, Cockcroft J, Bortel LV, et al. Non-invasive investigation of large arteries expert consensus document on arterial stiffness: Methodological issues and clinical applications. European Heart Journal. 2006;27:2588-2605.

6. Wang X, Keith JC, Jr., Struthers AD, Feuerstein GZ. Assessment of arterial stiffness, a translational medicine biomarker system for evaluation of vascular risk. Cardiovascular Therapeutics. 2008;26:214-223.

7. Williams B, Lacy PA, Yan P, et al. Development and validation of a novel method to derive central aortic systolic pressure from the radial pressure waveform using an $\mathrm{N}$-point moving average method. J. Am. Coll. Cardiol. 2011;57:951-961.

8. Stehouwer CDA, Henry RMA, Ferreira I. Arterial stiffness in diabetes and the metabolic syndrome: A pathway to cardiovascular disease. Diabetologia. 2008;51:527-539.

9. DeLoach SS and Townsend RR. Vascular stiffness: Its measurement and significance for epidemiologic and outcome studies. Clin. J. Am. Soc. Nephrol. 2008;3:184-192.

10. Chudleigh RA. How reliable is estimation of glomerular filtration rate at diagnosis of type 2 diabetes? Diabetes Care. 2007;30:300-305.

11. Rognant N. Performance of the chronic kidney disease epidemiology collaboration equation to estimate glomerular filtration rate in diabetic patients. Diabetes Care. 2011;34:1320-1322.

12. Stevens LA. Assessing kidney function - measured and estimated glomerular filtration rate. N. Engl. J. Med. 2006;354:2473-2483.

13. Williams B, Lacy PS, Yan P, et al. Development and validation of a novel method to derive central aortic systolic pressure from the radial pressure waveform using an $\mathrm{N}$-point moving average method. J. Am. Coll. Cardiol. 2011;57:951-961.
14. Nagai M, Komiya H, Mori Y, et al. Estimating visceral fat area by multifrequency bioelectrical impedance. Diabetes Care 2010;33:10771079.

15. Moe SM and Chen NX. Mechanisms of vascular calcification in chronic kidney disease. J. Am. Soc. Nephrol. 2008;19: 213-216.

16. JNavarro-González JF, Mora-Fernández C, de Fuentes MM, GarcíaPérez J. Inflammatory molecules and pathways in the pathogenesis of diabetic nephropathy. Nat. Rev. Nephrol. 2011;7:327-340.

17. Remuzzi G, Perico N, Macia M, Ruggenenti P. The role of reninangiotensin-aldosterone system in the progression of chronic kidney disease. Kidney International. 2005;68(99):S57-S65.

18. Schnermann J and Briggs JP. Tubuloglomerular feedback: mechanistic insights from gene-manipulated mice. Kidney International. 2008;74:418-426.

19. Toussaint ND and Kerr PG. Vascular calcification and arterial stiffness in chronic kidney disease: Implications and management. Nephrology. 2007;12:500-509.

20. Zee SVD, Baber U, Elmariah S, Winston J, Fuster V. Cardiovascular risk factors in patients with chronic kidney disease. Nat. Rev. Cardiol. 2009;6:580-589.

21. Nahas ME. Cardio-Kidney-Damage: A unifying concept. Kidney International. 2010;78:14-18.

22. Luk AOY. Metabolic syndrome predicts new onset of chronic kidney disease in 5,829 patients with type 2 diabetes: A 5-year prospective analysis of the Hong Kong diabetes registry. Diabetes Care.2008:31:2357-2361.

23. Bakris G. National kidney foundation consensus conference on cardiovascular and kidney diseases and diabetes risk: An integrated therapeutic approach to reduce events. Kidney International. 2010;78:726-736.

24. Schrijvers BF, De Vriese AS, Flyvbjerg A. From hyperglycemia to diabetic kidney disease: The role of metabolic, hemodynamic, intracellular factors and growth factors/cytokines. Endocr. Rev. 2004;25(6):971-1010.

25. Brownlee M. Biochemistry and molecular cell biology of diabetic complications. Nature. 2001; 414(6865):813-820.

26. Zarich SW. Antidiabetic agents and cardiovascular risk in type 2 diabetes. Nat. Rev. Endocrinol. 2009;5:500-506.

27. Ray KK, Seshasai SRK, Wijesuriya S, et al. Effect of intensive control of glucose on cardiovascular outcomes and death in patients with diabetes mellitus: Ameta-analysis of randomised controlled trials Lancet. 2009; 373: 1765-1772.

28. Fellstrom BC, et al. Rosuvastatin andcardiovascular events in patients undergoing hemodialysis. N. Engl. J. Med. 2009:360:1395-1407.

29. Baigent C, Landray MJ, Reith C, et al. SHARP Investigators. The effects of lowering LDL cholesterol with simvastatin plus ezetimibe in patients with chronic kidney disease (Study of Heart and Renal Protection): A randomised placebo-controlled trial. Lancet. 2011 ;377:2181-92.

30. Astor BC, Matsushita K, Gansevoort RT, et al. The chronic kidney disease prognosis consortium lower estimated glomerular filtration rate and higher albuminuria are associated with mortality and endstage renal disease: A collaborative meta-analysis of kidney disease population cohorts. Kidney International. 2011;79:1331-1340.

31. Haller H. Olmesartan for the delay or prevention of microalbuminuria in type 2 diabetes. N. Engl. J. Med. 2001;364:907917.

32. Asselbergs FW, Diercks GF, Hillege HL, et al. Effects of fosinopril and pravastatin on cardiovascular events in subjects with microalbuminuria. Circulation.110:2809-2816, 2004

33. Chudyk A. Effects of exercise on cardiovascular risk factors in type 2 diabetes: A meta-analysis. Diabetes Care. 2011;34:1228-1237.

34. Kronenberg F. Emerging risk factors and markers of chronic kidney disease progression. Nat. Rev. Nephrol. 2009;5: 677-689. 